\title{
Painéis Compensados Fabricados com Lâminas de Três Espécies de Eucaliptos
}

\author{
Sandra Kazmierczak ${ }^{1}$, Everton Hillig ${ }^{2}$, Setsuo Iwakiri ${ }^{3}$ \\ ${ }^{1}$ Programa de Pós-graduação em Ciências Florestais, Universidade Estadual do Centro-Oeste - UNICENTRO, \\ Irati/PR, Brasil \\ ${ }^{2}$ Universidade Estadual do Centro-Oeste - UNICENTRO, Irati/PR, Brasil \\ ${ }^{3}$ Universidade Federal do Paraná - UFPR, Curitiba/PR, Brasil
}

\section{RESUMO}

O objetivo foi analisar a qualidade de painéis compensados produzidos com lâminas de Eucalyptus saligna, Eucalyptus dunnii e o híbrido Eucalyptus urophylla $x$ Eucalyptus grandis, em diferentes composições de misturas. Foram produzidos painéis com $500 \times 500 \times 14 \mathrm{~mm}$ e sete camadas de lâminas, coladas com fenol-formaldeído (FF), na gramatura de $360 \mathrm{~g}$. $\mathrm{m}^{-2} \mathrm{em}$ linha dupla e teores de sólidos de $28 \%$ e $32 \%$. A prensagem dos painéis foi realizada a $130{ }^{\circ} \mathrm{C}, 12 \mathrm{kgf} . \mathrm{cm}^{-2}$ e 15 minutos. Analisaram-se as propriedades massa específica, umidade, absorção d'água, inchamento em espessura, cisalhamento na linha de cola e flexão estática. Os resultados foram comparados aos requisitos do catálogo técnico da ABIMCI e das normas EN 314-2 e DIN 68792. Os painéis de Eucalyptus dunnii apresentaram menores valores de propriedades mecânicas, com tendência de aumento quando em misturas. Os painéis de Eucalyptus saligna e do híbrido Eucalyptus urophylla $x$ Eucalyptus grandis apresentaram bons resultados, tanto puros como em mistura com as outras espécies.

Palavras-chave: Eucalyptus sp., laminação, painéis de madeira.

\section{Quality of Plywood Panels Made with Wood Veneer of Three Eucalyptus Species}

\begin{abstract}
The quality of plywood produced with wood veneer of Eucalyptus saligna, Eucalyptus dunnii and Eucalyptus urophylla $x$ Eucalyptus grandis hybrid in different compositions was analyzed. Seven-layer veneer panels measuring $500 \times 500 \times 14 \mathrm{~mm}$, glued with phenol-formaldehyde (PF), weighing 360 g. $\mathrm{m}^{-2}$ in double line and with 28 or $32 \%$ of solid content were produced. Panels were pressed under $130^{\circ} \mathrm{C}$ and $12 \mathrm{kgf} . \mathrm{cm}^{-2}$, during 15 minutes. We analyzed the properties of specific gravity, moisture content, water absorption, thickness swelling, glue line shear and static bending. The results were compared to the requirements of the ABIMCI technical catalog and EN 314-2 and DIN 68792 standards. Eucalyptus dunnii panels presented lower values of mechanical properties, with an upward trend when in mixtures. The panels produced with Eucalyptus saligna and the Eucalyptus urophylla $x$ Eucalyptus grandis hybrid showed good results, either pure or mixed with the other species.
\end{abstract}

Keywords: Eucalyptus sp., laminating, wood panels. 


\section{INTRODUÇÃO}

São vários os atributos que tornam importantes as mais diferentes espécies de eucalipto como fonte de matéria-prima fabril, dentre elas a sua capacidade produtiva, a adaptabilidade a diversos ambientes e a diversidade de espécies, possibilitando atender diversos segmentos da produção industrial madeireira (Assis, 1999).

De acordo com Interamnense (1998), os motivos mais relevantes que restringem a sua utilização para algumas finalidades são a existência de certas características indesejáveis, sendo as mais importantes a ocorrência de colapso durante a secagem e a presença das tensões de crescimento.

Guimarães et al. (2009) avaliaram 15 procedências advindas de espécies de Eucalyptus cloeziana, Eucalyptus grandis e Eucalyptus saligna, para confecção de painéis compensados multilaminados. Concluíram que o material estudado apresentou potencial para manufatura de painéis de madeira compensada.

Bortoletto (2003) estudou a produção de compensados fenólicos a partir da madeira de onze espécies de eucaliptos, para indicação dos seus possíveis usos em construção civil. Segundo o autor, o ensaio de cisalhamento na linha de cola avalia se o compensado pode ser destinado ao uso interior (avaliado no ensaio seco), intermediário (avaliado no ensaio úmido) ou exterior (avaliado no ensaio pós-fervura). Os resultados indicaram que os compensados de todas as espécies avaliadas, que incluem o E. saligna, podem ser destinados ao uso externo, com exceção para o compensado de E. citriodora, restrito aos usos interno e intermediário.

Almeida (2002) desenvolveu um estudo com o híbrido E. urophylla $\times$ E. grandis, cujo objetivo principal foi avaliar o potencial de utilização da madeira de dois clones para a produção de lâminas e de painéis compensados, detectando diferenças significativas para o fator clone em relação às propriedades dos painéis.

Hoje, sabe-se por experiência profissional que a espécie E. dunnii não é utilizada para confecção de painéis compensados, mas existe a necessidade de serem pesquisadas formas alternativas de seu uso. Iwakiri et al. (2007) concluíram sobre o potencial dessa espécie para a produção de painéis compensados estruturais. As espécies E. saligna e e o híbrido E. urophylla $x$ E. grandis, já adaptadas ao clima da região Sul, atualmente são usadas na produção de compensados, no entanto, carecem de estudos sobre a qualidade de seus painéis.

$\mathrm{O}$ E. saligna é uma árvore de tamanho alto a muito alto, atingindo 30 a $55 \mathrm{~m}$ de altura, com excelente forma do fuste e copa, que chega alcançar $1 / 2$ a $1 / 3$ da altura total da árvore. A distribuição natural da espécie situa-se entre as latitudes de 28 a $35^{\circ} \mathrm{S}$, em altitudes desde o nível do mar até $1.000 \mathrm{~m}$ (Mora \& Garcia, 2000). Segundo Ferreira (1979), a madeira do E. saligna está classificada como de média massa específica básica, entre 0,45 e 0,60 g. $\mathrm{cm}^{-3}$.

Nos estados do Paraná e de Santa Catarina, regiões propícias ao inverno rigoroso, com altitudes entre 500 e $1.000 \mathrm{~m}$, o E. dunnii tem demonstrado boa tolerância às geadas (Higa et al., 2000). No Brasil, sob condições de povoamentos implantados, sua madeira apresenta massa específica básica e composição química semelhante à de E. grandis, com teor de lignina ligeiramente inferior (Barrichelo \& Brito, 1976).

O híbrido E. urophylla $x$ E. grandis foi desenvolvido no Brasil com objetivo de obter plantas com melhor qualidade, bom crescimento e resistência a doenças. Segundo Costa (2011), as suas árvores formam lenho de maior massa específica básica em relação às árvores de suas espécies originais, em torno de $0,56 \mathrm{~g} . \mathrm{cm}^{-3}$, apresentando elevada taxa de crescimento volumétrico.

Dessa forma, este estudo trata da utilização das espécies Eucalyptus dunnii, Eucalyptus saligna e do híbrido Eucalyptus urophylla $x$ Eucalyptus grandis como fontes de matéria-prima para painéis compensados, tanto no seu uso isolado quanto em composições mistas.

\section{MATERIAIS E MÉTODOS}

\subsection{Modelo experimental}

Os painéis confeccionados variaram com os fatores composição de lâminas e teor de sólidos de adesivo, configurando assim um experimento fatorial incompleto. Foram utilizados três teores de sólidos de adesivo e seis composições de espécies. No entanto, por problemas técnicos na prensa, não foi possível produzir os painéis de três dos tratamentos com teor de sólido do adesivo de $36 \%$. Dessa forma, o arranjo fatorial foi incompleto, mas permitiu a análise dos três teores de sólido de adesivo e das seis composições de espécies, além da interação entre os fatores (ver item 2.5 Análise Estatística). A Tabela 1 apresenta a composição dos painéis nos diferentes tratamentos. 


\subsection{Laminação e secagem}

O material utilizado neste trabalho foi obtido de plantios na região Centro-Sul do Estado do Paraná. Foram colhidas duas árvores de cada espécie, sendo usadas as duas primeiras toras de cada árvore, com $1,85 \mathrm{~cm}$, para o processo de laminação.

Das extremidades de cada tora foram retirados discos, com cinco $\mathrm{cm}$ de espessura, para determinação da massa específica básica da madeira. Dos discos, foram retiradas duas cunhas opostas entre si e com ângulo de $30^{\circ}$, para uso na determinação da massa específica básica. Essa determinação foi realizada com base na Norma NBR 11941 (ABNT, 2003) empregando o método da balança hidrostática para obtenção do volume saturado.

As toras de E. dunnii, por serem originárias de reflorestamento não comercial, sem devidas práticas silviculturais, foram destinadas à vaporização, favorecendo o melhor acabamento nas superfícies das lâminas e facilitando no processo de laminação. $\mathrm{O}$ tratamento foi realizado em câmara apropriada, durante um período de 12 horas, a uma temperatura de 70 a $80^{\circ} \mathrm{C}$. As toras de E. saligna e do híbrido E. urophylla $\times$ E. grandis, obtidas de reflorestamento comercial, não foram submetidas ao processo de aquecimento, sendo laminadas logo após o efetivo corte e traçamento.

O processo de laminação das toras ocorreu em um torno laminador de marca Thons Benato, sendo processadas lâminas com dimensões de $1,70 \mathrm{~cm}$ de comprimento e espessura nominal de $2,2 \mathrm{~mm}$. A regulagem do torno teve ângulo de afiação da faca de $21^{\circ}$; ângulo da faca de $90^{\circ} 00^{\prime}$, variando automaticamente para $89^{\circ} 00^{\prime}$; abertura horizontal de 1,8 $\mathrm{mm}$ e abertura vertical de $0,45 \mathrm{~mm}$.

\subsection{Produção dos painéis de madeira compensada}

$\mathrm{O}$ adesivo utilizado foi à base de fenol-formaldeído com os parâmetros especificados na Tabela 2.

A Tabela 3 ilustra a proporção de adesivo e de extensor por teor de sólido. O preparo do adesivo teve mistura de resina com extensores de trigo e água, adaptando cada batida à formulação de teor de sólido para as três diferentes proporções.

Para cada teor de sólido ilustrado na Tabela 3 foram efetuadas três batidas de cola utilizando batedeira elétrica para homogeneização da resina com o extensor. Para a determinação da viscosidade, foi usado um copo Ford número 8 e um cronômetro, verificando-se que a viscosidade da cola ficou entre

Tabela 1. Composição dos painéis de madeira compensada.

Table 1. Composition of plywood panels.

\begin{tabular}{ccccccccc} 
Trat. & TS & Composição & Trat. & TS & Composição & Trat. & $\begin{array}{c}\text { Com- } \\
\text { posição }\end{array}$ \\
$\mathbf{1}$ & 28 & ES & $\mathbf{6}$ & 28 & ED/EU & $\mathbf{1 1}$ & 32 & $\mathrm{EU} / \mathrm{ES}$ \\
$\mathbf{2}$ & 28 & $\mathrm{ED}$ & $\mathbf{7}$ & 32 & $\mathrm{ES}$ & $\mathbf{1 2}$ & 32 & $\mathrm{ED} / \mathrm{EU}$ \\
$\mathbf{3}$ & 28 & $\mathrm{EU}$ & $\mathbf{8}$ & 32 & $\mathrm{ED}$ & $\mathbf{1 3}$ & 36 & $\mathrm{ES} / \mathrm{ED}$ \\
$\mathbf{4}$ & 28 & $\mathrm{ES} / \mathrm{ED}$ & $\mathbf{9}$ & 32 & $\mathrm{EU}$ & $\mathbf{1 4}$ & 36 & $\mathrm{EU} / \mathrm{ES}$ \\
$\mathbf{5}$ & 28 & $\mathrm{EU} / \mathrm{ES}$ & $\mathbf{1 0}$ & 32 & $\mathrm{ES} / \mathrm{ED}$ & $\mathbf{1 5}$ & 36 & $\mathrm{ED} / \mathrm{EU}$ \\
\hline
\end{tabular}

ES: E. saligna (capa e miolo); ED: E. dunnii (capa e miolo); EU: E. urophylla $x$ E. grandis (capa e miolo); ES/ED: E. saligna (capa) e E. dunnii (miolo); EU/ES: E. urophylla $x$ E. grandis (capa) e E. saligna (miolo); ED/EU: E. dunnii (capa) e E. urophylla $x$ E. grandis (miolo); Trat.: Tratamento; TS: Teor de sólidos do adesivo.

Tabela 2. Parâmetros técnicos do adesivo.

Table 2. Technical parameters of the adhesive.

\begin{tabular}{cc} 
PARAMETROS & RESINA FENOLICA \\
Teor de Sólido $\left(1 \mathrm{~g} / 3 \mathrm{~h} / 105^{\circ} \mathrm{C}\right)$ & $48-51 \%$ \\
Viscosidade Brokf $\left(25^{\circ} \mathrm{C}\right)$ & $400-800 \mathrm{cP}$ \\
$\mathrm{pH}\left(25^{\circ} \mathrm{C}\right)$ & $11,5-13,0$ \\
Gel Time & $6-11 \mathrm{minutos}$ \\
\hline Massa específica $\left(25^{\circ} \mathrm{C}\right)$ & $1,19-1,25 \mathrm{~g} / \mathrm{cm}^{3}$ \\
\hline
\end{tabular}


Tabela 3. Formulações das batidas de cola.

Table 3. Formulations beats glue.

\begin{tabular}{ccccc|} 
Teor de sólido (\%) & Adesivo $(\mathbf{k g})$ & Trigo $\mathbf{( k g )}$ & Água $(\mathbf{k g})$ & Total \\
\hline 28 & 5 & 2 & 1,75 & 8,75 \\
32 & 5 & 1,5 & 1,12 & 7,62 \\
36 & 5 & 1 & 0,75 & 6,75 \\
\hline
\end{tabular}

42 e 53 segundos. Para determinação da gramatura foi utilizada uma balança digital. A gramatura dos painéis foi de $360 \mathrm{~g}$. $\mathrm{m}^{-2}$ linha dupla.

Foram produzidos quarenta e cinco painéis de madeira compensada, com espessura de $14 \mathrm{~mm}$ e sete camadas de lâminas, de dimensões de $50 \times 50 \mathrm{~cm}$.

O processo de produção dos painéis seguiu a ordem de atividades: medição da gramatura, o espalhamento de cola, pré-prensagem e assemblagem, e finalizando com a prensagem a quente. $\mathrm{O}$ espalhamento da cola foi manual, com auxílio de uma espátula. O tempo de assemblagem e pré-prensagem foram entre vinte e vinte cinco minutos. Logo após, os painéis foram prensados à temperatura de $130{ }^{\circ} \mathrm{C}$, com $12 \mathrm{kgf}$. $\mathrm{cm}^{-2}$ de pressão e tempo de prensagem de 15 minutos, com tempo máximo de carregamento e calagem de dois minutos.

\subsection{Ensaios físicos e mecânicos}

Todos os ensaios foram realizados com base nas normas da ABNT para madeiras compensadas (ABNT, 1986a, b, c, d, e, 2006). Realizaram-se os ensaios físicos de teor de umidade, massa específica, absorção d'água, inchamento e inchamento mais recuperação em espessura e os ensaios mecânicos de flexão estática e cisalhamento na linha de cola.

Os ensaios mecânicos foram realizados em máquina universal de ensaios da marca EMIC, modelo DL 30000, eletromecânica, capacidade $300 \mathrm{kN}$. Neste trabalho calculou-se o inchamento e o inchamento mais recuperação da espessura, após a absorção de água pelo compensado. Para isso, utilizou-se a norma NRB 9535 (ABNT, 1986e), adaptada. Essa adaptação foi necessária, pois a equação de recuperação de espessura citada na referida norma não coincide com a definição dada na mesma norma. Assim, a equação de recuperação de espessura citada nesta norma foi usada para expressar o inchamento em espessura.

\subsection{Análise estatística}

A análise das propriedades físicas e mecânicas dos painéis foi realizada mediante análise de variância (ANOVA) fatorial incompleta, considerando os fatores misturas de espécies e teor de sólido da batida de cola, com $95 \%$ de probabilidade. Foi realizado o teste de Levene para homogeneidade de variâncias e a distribuição se aproximou da normal. Para análise da interação, foram considerados os 15 tratamentos. Para análise dos efeitos principais, foram considerados apenas os tratamentos que comporiam um modelo completo, ou seja, na análise das composições foram considerados os 12 tratamentos com os teores de sólido de $28 \%$ e $32 \%$, e para a análise dos teores de sólidos foram considerados os nove tratamentos com as composições mistas.

\section{RESULTADOS E DISCUSSÕES}

\subsection{Massa específica básica da madeira}

Os valores médios da massa específica básica da madeira de cada espécie são apresentados na Tabela 4 .

Segundo os parâmetros do Programa Nacional de Qualidade da Madeira - PNQM (ABIMCI, 2009), as toras com massa específica menor que $0,50 \mathrm{~g} . \mathrm{cm}^{-3}$ são classificadas na classe I, que não necessita cozimento. Walker (1993), citado por Almeida (2002), comenta que as espécies adequadas para laminação tendem a apresentar massa específica básica entre 0,38 e $0,70 \mathrm{~g} \cdot \mathrm{cm}^{-3}$. Assim, pode-se dizer que as espécies utilizadas apresentaram médias de massa específica básica de madeira dentro do recomendado para laminação.

\subsection{Qualidade das lâminas}

A Figura 1 ilustra as lâminas das diferentes espécies após a secagem. Verificou-se que todas as lâminas apresentaram boa qualidade, no entanto, as lâminas de E. dunnii, por serem provenientes de reflorestamento onde não foi realizada desrama, apresentaram uma quantidade considerável de nós. 
Tabela 4. Valores médios de massa específica básica por espécie.

Table 4. Mean values of basic density by species.

\begin{tabular}{cccc}
\hline Espécie & Idade (anos) & Meb $\left(\mathbf{g} \cdot \mathbf{~ c m}^{-3}\right)$ & DAP $(\mathbf{c m})$ \\
\hline E. saligna & 19 & 0,56 & 17,30 \\
\hline E. dunnii & 20 & 0,59 & 17,80 \\
\hline E. urophylla $x$ E. grandis & 12 & 0,49 & 16,37 \\
\hline
\end{tabular}

Meb: massa específica básica; DAP: diâmetro na altura do peito.
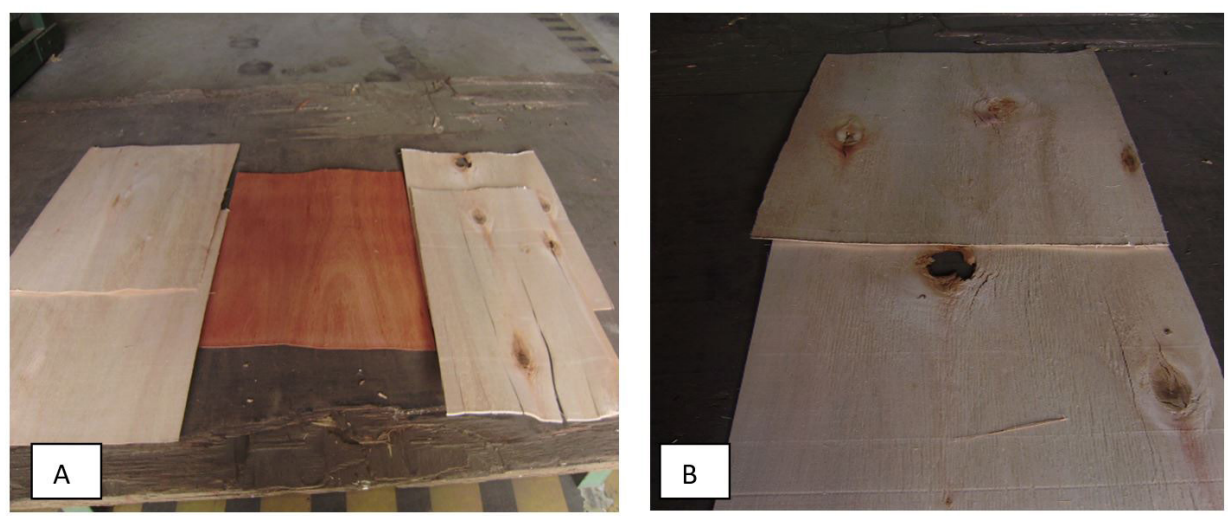

Figura 1. Qualidade das lâminas secas. (A) E. urophylla x E. grandis, E. saligna e E.dunnii; (B) E. dunnii. Figure 1. Quality dry veneer. (A) E. urophylla $x$ E. grandis, E. saligna and E.dunnii; (B) E. dunnii.

\subsection{Avaliação da estabilidade dimensional dos painéis}

As médias e o resultado da análise de variância dos efeitos dos fatores teor de sólidos e composição dos painéis, para ensaios de massa específica aparente, teor de umidade, absorção de água, inchamento em espessura e inchamento mais recuperação são apresentados na Tabela 5 .

A pressão de prensagem provoca a redução de espessura das lâminas individuais ou do próprio painel que elas compõem, bem como o aumento da massa específica do compensado em relação à da madeira que lhe originou. Isso explica o fato de os painéis apresentarem massa específica aparente (Mea) superior à esperada em função da madeira e do adesivo usado.

Confrontados os resultados encontrados para teor de umidade dos painéis (U\%), verifica-se que as médias encontram-se de acordo com o requisito máximo exigido pelo catálogo técnico do PNQM, que é de 18\% (ABIMCI, 2009).

Para o fator teor de sólidos, verificou-se que houve diferença estatística significativa entre as médias para inchamento em espessura (I). Para o fator composição, houve diferença entre as médias para as propriedades analisadas, exceto para absorção d'água e inchamento em espessura. Somente para IR a interação entre os fatores foi estatisticamente significativa.

Os resultados deste estudo para médias de inchamento em espessura e inchamento mais recuperação em espessura foram próximos aos encontrados no estudo realizado com E. viminalis e E. robusta por Keinert \& Interamnense (1994).

Com relação ao inchamento em espessura (I), os painéis com teor de sólido da batida de cola de $28 \%$ apresentaram melhor resistência ao inchamento. Entre as misturas, a composição que apresentou menor valor de I foi dos painéis produzidos com Eucalyptus dunnii, com valor médio inferior aos demais painéis. Esse fato foi atribuído à menor razão "Mea do painel / Meb da madeira", quando comparada com a apresentada pelos painéis produzidos com as demais composições. Assim, para esses painéis houve pouca redução da espessura das lâminas e menor redução de porosidade.

Quando se produziram painéis de E. dunni em mistura com E. saligna, esse efeito foi minimizado e os painéis apresentaram um valor médio intermediário 
Tabela 5. Médias das propriedades físicas dos painéis compensados em função dos fatores estudados (teor de sólido do adesivo e composição de espécies).

Table 5. Mean values of panels physical properties due to the factors studied (adhesive solid content and species composition).

\begin{tabular}{|c|c|c|c|c|c|c|}
\hline Fatores & Níveis & $\begin{array}{c}\text { Mea } \\
\left(\mathrm{g} \cdot \mathrm{cm}^{-3}\right)\end{array}$ & $\begin{array}{l}\text { TU } \\
(\%)\end{array}$ & $\begin{array}{l}\text { AA } \\
(\%)\end{array}$ & $\begin{array}{c}\mathrm{I} \\
(\%)\end{array}$ & $\begin{array}{l}\text { IR } \\
(\%)\end{array}$ \\
\hline \multirow{4}{*}{$\begin{array}{l}\text { Teor de sólido } \\
\text { do adesivo }\end{array}$} & $28 \%$ & $0,69 \mathrm{a}$ & $11,08 \mathrm{a}$ & $23,98 \mathrm{a}$ & $12,21 \mathrm{a}$ & $8,48 \mathrm{a}$ \\
\hline & $32 \%$ & $0,68 \mathrm{a}$ & 10,79 a & $23,97 \mathrm{a}$ & $14,38 \mathrm{~b}$ & $8,72 \mathrm{a}$ \\
\hline & $36 \%$ & $0,70 \mathrm{a}$ & 10,97 a & $24,63 \mathrm{a}$ & $13,41 \mathrm{ab}$ & $7,80 \mathrm{a}$ \\
\hline & Fcalc. & 0,51 & 0,83 & 0,49 & $5,45^{*}$ & 1,55 \\
\hline \multirow{7}{*}{$\begin{array}{l}\text { Composição } \\
\text { de espécies }\end{array}$} & ES & $0,82 \mathrm{~d}$ & $9,25 \mathrm{a}$ & $25,78 \mathrm{a}$ & $15,01 \mathrm{~b}$ & $7,20 \mathrm{ab}$ \\
\hline & ED & $0,67 \mathrm{bc}$ & $11,00 \mathrm{~cd}$ & $23,02 \mathrm{a}$ & $8,83 \mathrm{a}$ & $8,41 \mathrm{~b}$ \\
\hline & EU & $0,60 \mathrm{a}$ & $10,30 \mathrm{~b}$ & $23,15 \mathrm{a}$ & $13,43 \mathrm{~b}$ & $6,22 \mathrm{a}$ \\
\hline & ES/ED & $0,68 c$ & $10,94 \mathrm{~cd}$ & 24,90 a & $12,63 \mathrm{~b}$ & $10,25 c$ \\
\hline & EU/ES & $0,77 \mathrm{~d}$ & $1,59 \mathrm{bc}$ & $23,32 \mathrm{a}$ & $13,18 \mathrm{~b}$ & $7,81 \mathrm{~b}$ \\
\hline & $\mathrm{ED} / \mathrm{EU}$ & $0,62 \mathrm{ab}$ & $11,28 \mathrm{~d}$ & $23,70 \mathrm{a}$ & $14,09 \mathrm{~b}$ & $7,74 \mathrm{~b}$ \\
\hline & Fcalc. & $21,85^{\star}$ & $16,39^{*}$ & 1,91 & $11,21^{\star}$ & $9,22^{*}$ \\
\hline Interação & Fcalc. & 1,22 & 2,20 & 0,80 & 1,19 & $3,53^{*}$ \\
\hline
\end{tabular}

${ }^{*}$ F significativo para 5\% de probabilidade. Médias seguidas pela mesma letra não diferem estatisticamente pelo teste de Duncam. Mea: Massa específica aparente dos painéis compensados; TU: Teor de umidade dos painéis compensados; AA: Absorção d'água; I: Inchamento em espessura; IR: Inchamento mais recuperação em espessura; ES: E. saligna; ED: E. dunnii; EU: E. urophylla x E. grandis; ES/ED: E. saligna e E. dunnii; EU/ES: E. urophylla X E. grandis e E. saligna; ED/EU: E.dunnii e E. urophylla $x$ E. grandis.

de I, mas que não diferiu estatisticamente das demais composições.

Houve interação entre os fatores teor de sólido e composição do painel para IR. Dessa forma, realizouse uma análise da variação das médias de IR de cada teor de sólido em cada composição, que pode ser visualizada na Figura 2.

É possível verificar que todos os painéis fabricados em composições puras (ED, ES, EU) e mais a composição mista EU/ES apresentaram maior IR para um menor teor de adesivo, embora não na mesma proporção.

Por outro lado, para as composições mistas com lâminas de Eucalyptus dunnii, a variação de IR não seguiu essa lógica, sendo que com o aumento do teor de sólido de 28 para $32 \%$ ocorreu um aumento não significativo de IR. Verifica-se que há uma influência das características das lâminas de E. dunnii que interferem no IR, especialmente nas composições mistas, demonstrando que para essas lâminas o teor de sólido de $32 \%$ teve efeito negativo. Esse fato está relacionado às características anatômicas da madeira dessa espécie e à qualidade inferior das lâminas (presença de nós).

\subsection{Avaliação das propriedades mecânicas dos painéis}

As médias e o resultado da análise de variância dos efeitos dos fatores teor de sólido da batida de cola e composição dos painéis para ensaios de resistência ao cisalhamento na linha de cola e flexão estática são apresentados na Tabela 6 .

Os resultados indicaram que não houve influência dos diferentes teores de sólido da batida de cola nos valores das propriedades mecânicas dos painéis. Essa mesma constatação, importante do ponto de vista econômico, foi verificada por Iwakiri et al. (2007) para os valores de resistência da linha de cola de painéis compensados fabricados com lâminas de E. grandis e E. dunnii.

A análise do fator composição mostra que os painéis compostos por lâminas da espécie E. dunnii apresentaram menor valor de média para as propriedades mecânicas. Os painéis compostos por lâminas do híbrido E. urophylla $x$ E. grandis apresentaram maior valor de média para RLC e para falha na madeira, enquanto a composição pura de E. saligna apresentou maior valor de média para MOR e MOE. 


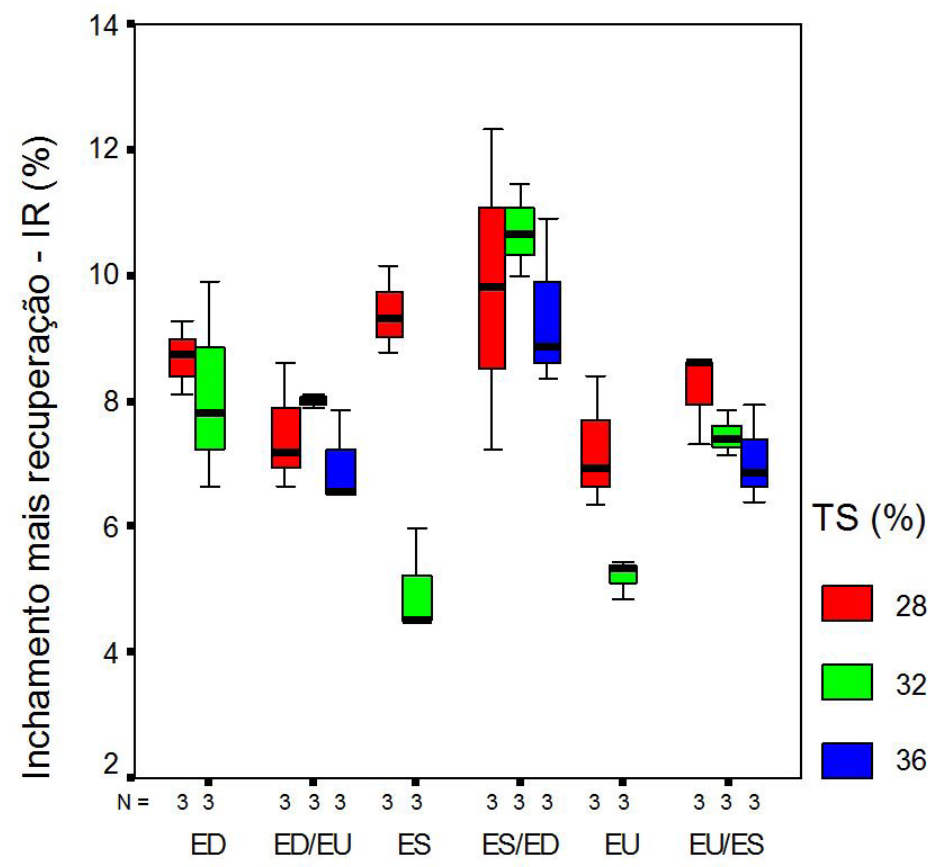

Composição do painel

Figura 2. Médias de Inchamento mais recuperação (IR) de cada teor de sólido em cada composição dos painéis. $\mathrm{TS}=$ Teor de sólido; $\mathrm{ES}=$ E. saligna $; \mathrm{ED}=E$. dunnii; $\mathrm{EU}=$ E. urophylla $\times$ E.grandis.

Figure 2. Recovery of thickness (IR) mean of each solids content in each plywood composition. TS = Solid Contents; $\mathrm{ES}=$ E. saligna; $\mathrm{ED}=$ E. dunnii; $\mathrm{EU}=E$. urophylla $x$ E. grandis .

Tabela 6. Médias das propriedades mecânicas dos painéis compensados em função dos fatores estudados (teor de sólido do adesivo e composição de espécies).

Table 6. Mean values of panel mechanical properties due to the factors studied (adhesive solid content and species composition).

\begin{tabular}{|c|c|c|c|c|c|}
\hline Fatores & Níveis & $\begin{array}{c}\text { RLC } \\
(\mathrm{MPa})\end{array}$ & $\begin{array}{l}\text { FM } \\
(\%)\end{array}$ & $\begin{array}{l}\text { MOR } \\
\text { (MPa) }\end{array}$ & $\begin{array}{l}\text { MOE } \\
(\mathrm{MPa})\end{array}$ \\
\hline \multirow{4}{*}{ Teor de sólidos } & 28 & $1,82 \mathrm{a}$ & $69,17 \mathrm{a}$ & $42,45 \mathrm{a}$ & $6776,29 a$ \\
\hline & 32 & $1,70 \mathrm{a}$ & $72,78 \mathrm{a}$ & $42,92 \mathrm{a}$ & $6023,12 \mathrm{a}$ \\
\hline & 36 & $1,41 \mathrm{a}$ & $68,06 \mathrm{a}$ & $43,08 \mathrm{a}$ & $6797,63 \mathrm{a}$ \\
\hline & Fcalc. & 0,78 & 0,62 & 0,02 & 1,27 \\
\hline \multirow{7}{*}{ Composição } & ES & $1,43 \mathrm{ab}$ & $65,00 \mathrm{~b}$ & $44,53 \mathrm{~b}$ & $7544,29 \mathrm{~b}$ \\
\hline & ED & $0,73 \mathrm{a}$ & $42,08 \mathrm{a}$ & $31,82 \mathrm{a}$ & $5696,23 \mathrm{a}$ \\
\hline & EU & $2,04 \mathrm{~b}$ & $83,75 \mathrm{c}$ & $42,89 \mathrm{bc}$ & $6274,58 \mathrm{ab}$ \\
\hline & $\mathrm{ES} / \mathrm{ED}$ & $1,35 \mathrm{ab}$ & 52,91 a & $36,57 \mathrm{ab}$ & 5799,86 a \\
\hline & EU/ES & $2,32 \mathrm{~b}$ & $82,91 \mathrm{c}$ & $48,60 \mathrm{c}$ & $7203,44 \mathrm{~b}$ \\
\hline & $\mathrm{ED} / \mathrm{EU}$ & $1,62 \mathrm{ab}$ & $77,08 \mathrm{c}$ & $42,89 \mathrm{bc}$ & $6195,82 \mathrm{ab}$ \\
\hline & Fcalc. & $3,12^{*}$ & $17,56^{*}$ & $4,22^{*}$ & $2,98^{*}$ \\
\hline Interação & Fcalc. & 0,78 & 0,82 & 1,29 & 1,86 \\
\hline
\end{tabular}

${ }^{*}$ Significativo ao nível de $5 \%$ de probabilidade. Médias seguidas pela mesma letra não diferem estatisticamente entre si teste de Duncam. RLC: Ruptura na linha de cola; FM: Falha na madeira; MOR: Módulo de ruptura em flexão estática; MOE: Módulo de elasticidade em flexão estática; ES: E. saligna; ED: E. dunnii; EU: E. urophylla $x$ E. grandis. ES/ED; E. saligna e E. dunnii; EU/ES: E. urophylla $x$ E. grandis e E. saligna; ED/EU: E. dunnii e E. urophylla $x$ E. grandis. 
Os conceitos apresentados por Marra (1992) podem explicar por que os painéis de E. dunnii, produzidos com lâminas mais densas, apresentaram valores inferiores de resistência da linha de cola. A madeira mais densa apresenta menor porosidade, prejudicando as ações de mobilidade do adesivo e o processo de "ancoragem" entre a linha de cola e as lâminas adjacentes. Além disso, as lâminas de madeira dessa espécie apresentaram qualidade inferior às demais.

Em geral, os valores médios comparados com os encontrados na literatura foram satisfatórios para RLC, atendendo ainda aos requisitos mínimos exigidos pela norma europeia EN 314-2 (European Standard, 1996). Para o MOR e o MOE, as médias foram satisfatórias, comparadas ao requisito mínimo exigido pela norma DIN 68792 (DIN, 1979), especificados para o uso de painéis em forma de concreto, exceto os painéis produzidos com lâminas da espécie E. dunnii, que apresentaram médias de MOR inferiores ao estabelecido pela norma.

\section{CONCLUSÕES}

$\mathrm{Na}$ estabilidade dimensional, os painéis produzidos com lâminas de madeira de Eucalyptus dunnii tiveram destaque, devido ao menor inchamento em relação às outras composições.

O fator composição dos painéis influenciou todas as propriedades mecânicas analisadas, sendo que, de forma geral e nas condições deste trabalho, verificou-se um desempenho inferior das lâminas da espécie Eucalyptus dunnii.

Os valores das propriedades de cisalhamento da linha de cola, MOR e MOE foram superiores para painéis de Eucalyptus saligna puros ou em misturas.

Os painéis produzidos com lâminas de Eucalyptus saligna e do híbrido Eucalyptus urophylla $x$ Eucalyptus grandis apresentaram bons resultados tanto no uso individual como em mistura entre eles e com Eucalyptus dunnii.

\section{STATUS DA SUBMISSÃO}

Recebido: 16 set., 2014

Aceito: 7 set., 2016

\section{AUTOR(ES) PARA CORRESPONDÊNCIA}

\section{Everton Hillig}

Universidade Estadual do Centro-Oeste UNICENTRO, BR 153, Km 07, Riozinho, CEP 84500-000, Irati, PR, Brasil e-mail: hillig@hotmail.com

\section{REFERÊNCIAS}

Almeida RR. Potencial da madeira de clones do híbrido Eucalyptus grandis $\times$ Eucalyptus urophylla para produção de lâminas e manufaturas de painéis compensado [dissertação]. Piracicaba: Escola Superior de Agricultura "Luiz de Queiroz", Universidade de São Paulo; 2002.

Assis TF. Aspecto do melhoramento de Eucalyptus para obtenção de produtos sólidos de madeira. In: Anais do Workshop Técnicas de Abate, Processamento e Utilização da Madeira de Eucalipto; 1999; Viçosa. Viçosa: DEF/SIF/ UFV/IEF; 1999. p. 61-72.

Associação Brasileira da Indústria da Madeira Processada Mecanicamente - ABIMCI. Programa Nacional da Qualidade da Madeira: compensados de madeira tropical. Curitiba: ABIMCI; 2009.

Associação Brasileira de Normas Técnicas - ABNT. NBR9484: compensado: determinação do teor de umidade. Rio de Janeiro; ABNT; 1986a.

Associação Brasileira de Normas Técnicas - ABNT. NBR9485: compensado: determinação da densidade. Rio de Janeiro; ABNT; 1986b.

Associação Brasileira de Normas Técnicas - ABNT. NBR9486: compensado: determinação da absorção de água. Rio de Janeiro; ABNT; 1986c.

Associação Brasileira de Normas Técnicas - ABNT. NBR9533: compensado: determinação da resistência à flexão estática. Rio de Janeiro; ABNT; 1986d.

Associação Brasileira de Normas Técnicas - ABNT. NBR-9535: compensado: determinação da em espessura $e$ inchamento mais recuperação em espessura. Rio de Janeiro; ABNT; 1986e.

Associação Brasileira de Normas Técnicas - ABNT. NBR11941: madeira: determinação da densidade básica. Rio de Janeiro; ABNT; 2003.

Associação Brasileira de Normas Técnicas - ABNT. NBRISO 12466-1: madeira compensada: qualidade de colagem. Parte 1: métodos de ensaio. Rio de Janeiro; ABNT; 2006.

Barrichelo LEG, Brito JO. A madeira das espécies de eucalipto como matéria-prima para a indústria de celulose e papel. Brasília: PRODEPEF; 1976.

Bortoletto G Jr. Produção de compensados com 11 espécies do gênero Eucalyptus, avaliação das suas propriedades 
físico-mecânicas e indicações para utilização. Scientia Forestalis 2003; 63: 65-78.

Costa JA. Qualidade da madeira de E. urograndis plantado no Distrito Federal, para a produção de celulose Kraft. [dissertação]. Brasília: Universidade de Brasília; 2011.

Deutsches Institut für Normung - DIN. DIN 68792: large are stuttering panels of veneer plywood for concrete and reinforced concrete. Berlin: DIN; 1979.

European Standard. EN 314-2: colagem do compensado de madeira: qualidade. Parte 2: exigências de teste. Bruxelas; 1996.

Ferreira, M. Escolha das espécies de eucaliptos. São Paulo: Circular técnica IPEF; 1979.

Guimarães JB Jr, Mendes LM, Mendes RF, Mori FA. Painéis compensado de eucalipto: estudo de caso de espécies e procedências. Cerne 2009; 15(1): 10-18.

Higa RCV, Higa AR, Trevisan R, Souza MVR. Resistência e resiliência a geadas em Eucalyptus dunnii Maiden Plantados em Campo do Tenente, PR. Boletim de Pesquisa Florestal 2000; 40: 67-76.
Interamnense MT. Utilização das madeiras de Eucalyptus cloeziana (F. Muell), Eucalyptus maculata (Hook) e Eucalyptus punctata DC var. punctata para produção de painéis compensados [dissertação] Curitiba: Setor de Ciências Agrárias, Universidade Federal do Paraná; 1998.

Iwakiri S, Keinert S Jr, Prata JG, Rosso S. Produção de painel compensado estrutural de Eucalyptus grandis e Eucalyptus dunnii. Floresta 2007; 37(3): 363-367. http:// dx.doi.org/10.5380/rf.v37i3.9932.

Keinert S Jr, Interamnense MT. Laminação, produção e testes de compensados a partir de Eucalyptus spp. Curitiba: CNPq; 1994. Relatório.

Marra AA. Technology of wood bonding. New York: Van Nostrand Reinhold; 1992.

Mora AL, Garcia CH. A cultura do eucalipto no Brasil. São Paulo: SBS; 2000.

Walker JCF. Primary wood processing: principles and practice. London: Chapman \& Hall; 1993. 